\title{
Learning Object Meta-data for a Database of Primary and Secondary School Resources
}

\author{
Daniel D. Suthers ${ }^{1}$, Susan Marie Johnson ${ }^{2}$ and Beth Tillinghast ${ }^{2}$ \\ ${ }^{1}$ Department of Information and Computer Sciences, University of Hawai i at Manoa, \\ Honolulu, HI, USA, ${ }^{2}$ Library and Information Sciences Program, University of \\ Hawai i at Manoa, Honolulu, HI, USA
}

\begin{abstract}
The learning object meta-data (LOM) is an emerging standard for annotation of educational entities (digital or non-digital) that are relevant to technology-supported learning. The annotations describe educational, legal, and technical characteristics of these resources. Development of this standard is sponsored by the IEEE Learning Technology Standards Committee. In this paper, we describe an application of the LOM to the construction of a database of resources available to schools in Hawai $i$ and report on both successes and issues encountered. Recommendations are made concerning modifications to the LOM and adoption of the LOM by others working in primary and secondary school contexts.
\end{abstract}

\section{INTRODUCTION}

Internet technology for learning, including Web-based resources, networked groupware and remote sensing have the potential to bring teachers and students together with a greater diversity and quantity of human, natural and technological resources than was previously possible. Educators and students can now access an enormous variety of Web-based expository materials, images, activity plans, simulations, etc., and interact with people from many walks of life over the Internet. Already pressed for time, how will educators sort through this cornucopia of information and misinformation and find the resources appropriate for the educational needs of their students? Clearly, in order to leverage the great potential of this de-facto world wide

Correspondence: Daniel D. Suthers, Department of Information and Computer Sciences, University of Hawai i at Manoa, 1680 East West Road, POST 303A, Honolulu, HI 96822, USA. E-mail: suthers@hawaii.edu 
digital library, educators will need help. This paper is concerned with one form of help: databases of meta-data or information that describes the relevant characteristics of educational resources sometimes called learning objects. Properly constructed meta-data databases that have interfaces designed to match educator's perspectives should enable them to find relevant learning objects more quickly.

There are two other factors that also motivate this work. In the United States, there is currently a strong emphasis on systemic reform in public school education at the primary and secondary school levels. Being systemic, this movement is encouraging and compelling a greater diversity of stakeholders to collaborate in their mutual interest in supporting achievement of high standards in the schools. For example, the Educational System Reform (http://www.ehr.nsf.gov/EHR/ESR/) division of the United States National Science Foundation requires that proposals for funding show evidence of significant collaborations between schools, universities and colleges, business and industry, and other community members in genuine support of sustainable reform (i.e., reform that continues beyond the funded period). As a result, organizations and individuals who have not previously worked together need to become aware of the resources they offer to each other. Hence, databases of resources that are tailored for particular locations are needed. The present work is one example of such a database.

A third motivating factor is economic. The cost of building educational materials, particularly technology-supported materials such as software, is high. All too often, persons and groups who are intellectually prepared to develop innovative new approaches to the application of technology to education spend most of their time rebuilding basic functionality. Recent interest in educational object repositories and educational technology standards is motivated in large part by the desire to be able to find and reuse the work of others. Some standards are being developed to describe learning objects so that they may be found (Milstead \& Feldman, 1999b). Others are being developed to facilitate the interoperability of these objects once they are found (Miller, 2000). This work is concerned primarily with standards for describing learning objects so that they may be found. Software interoperability has been addressed elsewhere (Koedinger et al., 1999; Roschelle et al., 1999). Standards for describing learning objects also address economic issues surrounding resource databases because databases are expensive to build. Rather than replicate existing meta-data, it is preferable to access existing meta-data repositories. However, this requires standard forms for meta-data. 
In summary, the demands of systemic reform and economic considerations require educators and their partners to be aware of the diversity of resources that are potentially available to them and to understand the significance or potential utility of these resources with respect to educational objectives. Resource databases should adequately describe a diverse variety of resources, yet relate them all to common educational objectives, describe the resources in terms understandable to educators, and interoperate with other major repositories. In this paper, we report on our first efforts to design such a resource database to meet these needs within the State of Hawai $i$ as part of a systemic initiative known as Hawai i Networked Learning Communities. Specifically we report on our use of an emerging standard, the learning object meta-data (LOM). The paper provides a brief introduction to the LOM, describes its application to HNLC, and discusses limitations and extensions to the LOM that were required. Finally, readers are provided with information on how to participate in the development of the LOM.

\section{BACKGROUND}

\section{Learning Object Meta-data}

Meta-data, simply defined, is data about data (Milstead \& Feldman, 1999a; Wason, unpublished). Meta-data defines the characteristics of other data so that it may be interpreted and used intelligently. In this sense meta-data enables us to use data as information. The phrase learning object is used to inclusively denote a wide variety of entities used to support learning, including but not limited to digital resources, such as software, multimedia, or hypertext, and nondigital resources such as courses of study, professional development programs, or persons who have volunteered to serve as mentors. Assembling these concepts, we come to LOM, which is somewhat of a misnomer in that the meta-data is not only describing data, but also other entities that are not data (such as persons). Yet the term 'meta-data' is already in wide use for this purpose, so will be used herein.

\section{Technical Standards}

A technical standard is a specification of shared terms, interfaces, representations, practices, etc. If an artifact (such as computer or networking hardware, a software program, or data representations) is constructed to be compliant with a technical standard, then that standard ensures that multiple stakeholders will 
be able to interpret or interface with that artifact without needing to ask for help from the creator of the artifact, i.e. a standard helps to ensure interoperability (Miller, 2000) and reuse. A standard is expressed in a document that sets forth the scope and purpose of the standard and the mandatory conditions for compliance. The existence of a standard, for example, for learning technologies, does not mean that everyone is expected to comply with the standard. It only sets forth the conditions for those who elect to claim compliance with the standard.

\section{The IEEE LTSC Learning Object Meta-data}

The Institute of Electrical and Electronics Engineers (IEEE), http:// www.ieee.org/) is an international organization for engineers of electrical and information technologies. IEEE has a well-defined standards development process administered by its Standards Activity Board (http://www.computer. org/standards/). The Learning Technology Standards Committee (LTSC), which was founded in 1996 by a group of academic, government, and industry representatives (including the author), chose to use the IEEE standards process for these reasons. The LTSC is an umbrella organization that sponsors approximately 20 learning technology standards efforts, at various levels of maturity ranging from speculative to approaching balloting. Examples include the Architecture and Reference Model, CBT Interchange language, and Learner Model, as well as the LOM. The LOM draft standard (also known by its IEEE identifier as 1484.12) is arguably the most mature of the LTSC draft standards. According to a proposed revision to the Project Authorization Request, "The purpose of this standard is to facilitate search, evaluation, acquisition, and use of learning objects, for instance by learners or instructors. The purpose is also to facilitate the sharing and exchange of learning objects, by enabling the development of catalogs and inventories, taking into account the diversity of cultural and lingual contexts in which the learning objects and their meta-data will be exploited."

The LOM standard is meant to provide a semantic model for describing properties of the learning objects themselves, rather than detailing ways in which these learning objects may be used to support learning. The LOM indicates the legal values and informal semantics of the meta-data elements, their dependencies on each other, and how they are composed into a larger structure. It is intended to be extended, and in fact a structure has been provided specifically for the purpose. The LOM is agnostic concerning 
bindings or implementations of meta-data in particular representations or notations, such as XML. (At this writing, a study group is exploring a separate XML binding specification.) No particular representation or implementation is specified or implied by the LOM. Systems that are LOM compliant may present users with any interface they wish and store the meta-data however they wish. The LOM specifies only the semantics of the meta-data in order to enable meaningful interchange of meta-data between systems.

An outline of the LOM meta-data elements as of draft 4.1 (IEEE Std. 1484.12, draft) is provided in Table 1. In this table, nesting indicates a compositional relationship. For example (adopting notation commonly used in the LOM committee), a single 1.3: Catalog.Entry consists of a 1.3.1: Catalogue and an 1.3.2: Entry; while a 9: Classification consists of several types of sub-elements, some of which themselves also have internal structure. Much important information has been left out of this table for space considerations. For example, some data elements may take on multiple values which may be ordered or unordered, and some must be taken from restricted vocabularies or reference other standards for their values.

Brief descriptions of the major element categories follow. 1: General provides information such as title, a brief textual description, and keywords. 2: Life.Cycle describes the development and current state of the resource. 3: Metameta.Data describes the meta-data itself, for example, who entered or validated this meta-data instance and what language it is written in. 4: Technical provides information on media type, size, software requirements, etc. for those learning objects to which these attributes apply. 5: Educational is intended to provide basic information about the pedagogical characteristics of the resource. This category includes some of the most controversial elements, to be discussed further below. 6: Rights describes the conditions under which one may acquire and use the learning object. 7: Relation is intended to describe the learning object in relation to other learning objects. At this writing there is a controversy concerning whether this may be used to control sequencing of a collection of learning objects, or whether that should be deferred to other standards being developed for the purpose. 8: Annotation allows for the accumulation of comments by persons who have used or are otherwise evaluating the learning object. 9: Classification provides a means of extending the LOM to meet specialized needs. This comes in the form of a generic structure for classifying the learning object in one or more taxonomic systems external to the LOM. Most of our extensions used 9: Classification. 
Table 1. Outline of Learning Object Meta-data Elements.

\begin{tabular}{|c|c|}
\hline & \\
\hline 1: General & 4.5: Installation Remarks \\
\hline 1.1: Identifier & 4.6: Other Platform Requirements \\
\hline 1.2: Title & 4.7: Duration \\
\hline 1.3: Catalog.Entry & \\
\hline 1.3.1: Catalogue & 5: Educational \\
\hline 1.3.2: Entry & 5.1: Interactivity.Type \\
\hline 1.4: Language & 5.2: Learning. Resource.Type \\
\hline 1.5: Description & 5.3: Interactivity Level \\
\hline 1.6: Keywords & 5.4: Semantic.Density \\
\hline 1.7: Coverage & 5.5: Intended.End.User.Role \\
\hline 1.8: Structure & 5.6: Context \\
\hline 1.9: Aggregation Level & 5.7: Typical.Age.Range \\
\hline & 5.8: Difficulty \\
\hline 2: Life.Cycle & 5.9: Typical.Learning.Time \\
\hline 2.1: Version & 5.10: Description \\
\hline 2.2: Status & 5.11: Language \\
\hline 2.3: Contribute & \\
\hline 2.3.1: Role & 6: Rights \\
\hline 2.3.2: Entity & 6.1: Cost \\
\hline 2.3.3: Date & 6.2: Copyright and Other Restrictions \\
\hline & 6.3: Description \\
\hline 3: MetaMeta.data & 7: Relation \\
\hline 3.1. Catalog Entry & 7.1: Kind \\
\hline $\begin{array}{l}\text { 3.2: Catalog Entry } \\
\text { 3.2.1: Catalogue }\end{array}$ & 7.2: Resource \\
\hline 3.2.2: Entry & 7.2.1: Identifier \\
\hline 3.3: Contribute & 7.2.2: Description \\
\hline 3.3.1: Role & 7.2.3: Catalog.Entry \\
\hline 3.3.2: Entity & 8: Annotation \\
\hline 3.3.3: Date & 8.1: Person \\
\hline 3.4: Meta-data Scheme & 8.2: Date \\
\hline 3.5: Language & 8.3: Description \\
\hline 4: Technical & 9: Classification \\
\hline 4.1: Format & 9.1: Purpose \\
\hline 4.2: Size & 9.2: Taxon.Path \\
\hline 4.3: Location & 9.2.1: Source \\
\hline 4.4: Requirements & 9.2.2: Taxon \\
\hline 4.4.1: Type & 9.2.2.1: Id \\
\hline 4.4.2: Name & 9.2.2.2: Entry \\
\hline 4.4.3: Minimum Version & 9.3: Description \\
\hline 4.4.4: Maximum Version & 9.4: Keywords \\
\hline
\end{tabular}




\section{HNLC RESOURCE DATABASE}

The remainder of this paper describes our first prototype design and implementation of a learning object resource database, specifically focusing on the use of the LOM as a guiding framework for the design, and on ways in which extensions to the LOM were required. We briefly describe the initiative that this database was intended to serve before discussing the application of the LOM itself.

\section{Hawai i Networked Learning Communities}

The Hawai `i Networked Learning Communities (HNLC, http://lilt.ics. hawaii.edu/hnlc/) initiative is a partnership between the Hawai i Department of Education (HDOE), the University of Hawai i, and many other stakeholders in the quality of Hawai i public education, such as business and nonprofit interests. HNLC's purpose is to prepare all students in Hawai i's public schools for life and careers in today's world by enabling them to attain high standards in science, math, engineering and technology (SMET) education. The HNLC initiative is supporting HDOE in its systemic standards-based reform efforts by leveraging Hawai i's rich land, sea, space, and cultural resources. A theme of "global environmental studies, situated locally" pervades the work. From the standpoint of technology-supported learning, HNLC has three major thrusts. First, professional development will help educators make better use of technologies as educational resources in their classrooms. Second, distance collaboration and remote sensing technology will bridge the distances between small rural schools and the islands' rich resources, enabling virtual access to field sites, research laboratories or equipment, and, most importantly, peers and mentors of students, teachers and others involved in the educational process. Third, a web-accessible database will address one of the most frequent requests encountered during our needs assessment: knowing what resources are available to educators in Hawai $\mathrm{i}$. This paper is about the suitability of the LOM for this database.

\section{Scope of the Database}

The database describes resources for public school education ranging from US grades Kindergarten $(\mathrm{K})$ to 12 , also abbreviated as K-12. Standards-based reform is essential to the initiative: hence all resources must be described with respect to the Hawai i Content and Performance Standards 
(HCPS, http://www.hcps.k12.hi.us/), a document specifying what should be taught and how students' learning should be assessed. A wide variety of resources will be described, making this a particularly challenging test implementation of the LOM. For example, the following resources might be included.

- A university program in which Ph.D. students have their expenses paid in exchange for mentoring teachers for a certain number of hours a month. This can take place over the Internet; ideally, the teacher's students become involved in field report in support of the Ph.D. thesis.

- Nationally recognized curricular resources developed at the University's Curriculum Research and Development Group. (http://www.hawaii.edu/ (rdg/)

- A software program with which students can construct explicit visual models of their evidential reasoning while participating in investigations (http://lilt.ics.hawaii.edu/belvedere/index.html)

- A network of autonomous weather stations and remote controlled cameras, to be placed in the Alaka'i swamp (one of the rainiest place on Earth) or Volcano National Park, in some cases with the cameras trained on individuals of endangered plant species, with radio links to the Internet (http://www.botany.hawaii.edu/pods/)

- A nurse practitioner at a local military hospital who volunteered her time to telementor students on medical topics.

- Malama Hawai $i$, a new environmental education project started by the famed Polynesian voyager Nainoa Thompson. (http://www.malamahawaii. org/)

- Advanced placement courses in computer science and discrete math, offered by our department to high school students via Hawai i DOE's Internet-based E-School (http://atr.k12.hi.us/eschool/index.shtml)

- The He'eia Ahupua'a, in which researchers and school children collaborate to study the integration of modern and traditional Hawai ian land management techniques (Internet collaboration and mentoring is being planned. (http://kauila.k12.hi.us/ ahupuaa/)

- A Community College's research grade 24 in. telescopes, recently displaced from Haleakala by larger telescopes and now being installed for Webaccessible use at the $\mathrm{CC}$. The telescopes are still viable for new asteroid, comet and supernova survey research that can be conducted by high school students over the web, being supervised by college students and their professional mentors. 
- Diverse resources for teaching constructed by teachers and made available to others as part of a new product-oriented approach to professional development credits being implemented by HDOE.

All of these fall within LOM's scope of "any entity, digital or non-digital, which can be used, re-used or referenced during technology supported learning" (from the original Project Authorization Request, http://ltsc.iee.org/parlo.htm) because we will be using distance collaboration and remote sensing technology (as well as the database itself) to support learning using these resources. To control the scope of our work, HNLC will prioritize the description of local resources and interface with other repositories for nationally available resources (e.g., GEM).

\section{HNLC LOM META-DATA}

In designing the meta-data for resources such as those listed above, we found it necessary to extend the LOM. As previously noted, the LOM is intended to be extended. In some cases the predefined LOM elements were adequate, and in other cases we were able to perform the desired extensions using the LOM 9: Classification facility. However, in a few cases it was necessary to extend restricted vocabularies (which is not normally allowed), and in other cases structural issues arose. In this section we describe the most significant extensions, including the issues just mentioned.

\section{Method}

Our team consisted of the authors along with Laura Girardeau (an Environmental Education graduate), and David Nickles (a Computer Science graduate).

Initially Johnson and Tillinghast wrote informal textual descriptions capturing the important information about a representative sample of the resources that we wanted to describe. After reviewing these descriptions Suthers presented the LOM draft 4.1 (IEEE Std. 1484.12, draft) to the entire team, which required extensive discussions for clarification. We then went through the textual descriptions and identified LOM elements in which the information expressed could be captured. Where we failed to find LOM elements for an item of information we extended the LOM, either by expanding on the vocabulary of an existing element or by creating an entirely 
new element under 9: Classification. Where new elements were needed we searched other repositories to find meta-data that we could use. Several iterations were required to understand the LOM structure well enough to define our instances of 9: Classification. (It should be noted that end users are not expected to understand the LOM: the LTSC community expects that suitable interfaces will be developed, and no end user will even need to know that the LOM exists. We were approaching the LOM as information professionals, not end users.) Then, Nickles created a Filemaker implementation of the resulting HNLC-LOM and provided the others with an interface for building meta-data (Fig. 2). Meta-data for our sample was then created by Johnson and Tillinghast. Suthers then reviewed the result to detect possible misunderstandings and issues. Suthers also compiled a first draft of issues and recommendations. This draft was shared with the LTSC-LOM committee, both via email and subsequently face to face in an LTSC meeting (Montreal, June 2000). Thanks to their feedback, many issues were resolved or reunderstood as non-issues, and many further clarifications resulted.

\section{Vocabularies}

The data type of LOM elements may be primitive (e.g., a string), reference other standards (e.g., vCard), or consist of a controlled vocabulary. In the latter case, the vocabulary may be restricted, meaning that only the terms listed may be used, or open with recommended practice, meaning that one should attempt to use one of the terms listed as the recommended practice but may extend this vocabulary if needed. One extends the vocabulary by using a tuple of form (See Classification, term). The term is the new term being added to the vocabulary. One must define an instance of 9: Classification that has the same 9.1: Purpose as the data element being extended, and define a 9.2: Taxon.Path as needed to indicate where the term falls within the taxonomic system indicated by 9.2.1: Source. (A taxon path can be thought of as a sequence of taxons, which begins at the root of a taxonomic hierarchy and works its way down the tree through intermediate nodes to the leaf node under which the object is being classified.)

For example, suppose one wants to extend 5.2: Learning.Resource.Type with the term "Curriculum" taken from the Gateway to Educational Materials (GEM) Resource Type vocabulary, (http://www.geminfo.org/Workbench/ Metadata/Vocab_Type.html). One would place the tuple (See_Classification, 'Curriculum') in the 5.2 location, and then construct an instance of 9: Classification with 9.1: Purpose=Learning.Resource.Type, a single 


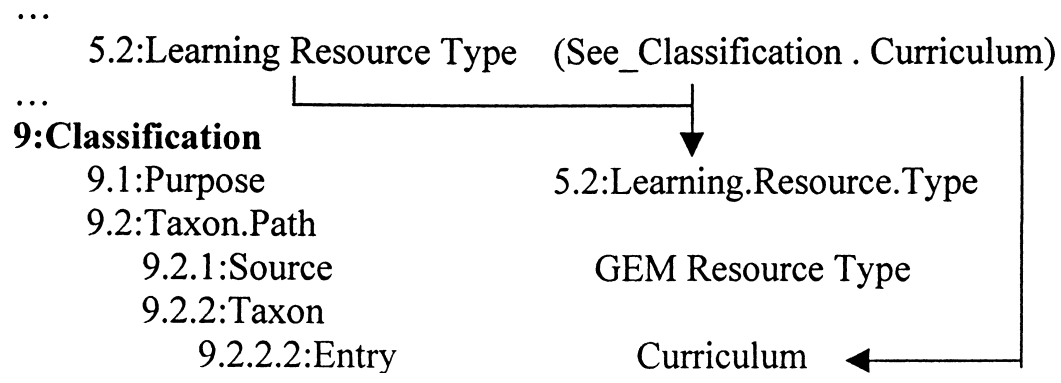

Fig. 1. Extending vocabularies with classification.

9.2: Taxon.Path with 9.2.1: Source $=$ 'GEM Resource Type,' and a single 9.2.2: Taxon with 9.2.2.2: Entry = 'Curriculum' (there is no ID available). As shown by the arrows in Figure 1 this arragnement would enable software to look up the appropriate entry in 9: Classification. Although this seems much more awkward than simply using the term 'Curriculum' in the 5.2: Learning. Resource.Type field, two points should be kept in mind. First, it is a powerful general-purpose way of extending vocabularies with information about the taxonomic source of the term, and hence its semantics. If we were to simply add a term to 5.2: Learning.Resource.Type its semantics would be inaccessible as there would be no place to record where the term came from. Second, the LOM information structures are neither specifications of an implementation nor specifications of a user interface: implementations are free to reorgainze the presentation of information to the user as convenient (e.g., to present extensions to vocabularies as if they were simply added to the same field in question).

We found several of the LOM vocabularies to be insufficient for our purposes. In one case, 5.2: Learning Resource Type, the vocabulary was open and the insufficiencies could be addressed via the extension mechanism just described. However, vocabularies for 1.9: Aggregation.Level, 5.1: Interactivity.Type (values: Active, Expositive. Mixed, or Undefined) and 5.5: Intended. End.User.Role (Teacher, Author, Learner, Manager) are restricted vocabularies, so cannot be extended in this way.

For example, the restricted vocabulary 1.9: Aggregation.Level has four values: 0: smallest level of aggregation (e.g., raw media data or fragments); 1 : collection of atoms (e.g., an HTML document with embedded pictures); 2: collection of level 1 resources (e.g., a web of HTML documents); 3: largest 
level of granularity (e.g., a course). Yet many of our resources are larger aggregations than courses, or bear upon such larger aggregations. The most obvious example is a curriculum designed to be used across multiple grade levels; another example is the Hawai i e-school which offers many on-line courses; and another is the Hawai i DOE's Strategic Plan (available at http:// www.k12.hi.us/). These resources range from curricular to systemic. Suppose we were forced to compress our levels, for example, using 0: raw media; 1 : a combination of the former $1 \& 2$, i.e., a document, a web, etc.; 2 : a course, and 3: curricular/systemic. The benefit of the precise vocabulary would be lost if this data migrated to a context using the assignments suggested by the examples given above and in the LOM. It would be preferable to add 4: curriculum and 5: systemic resource.

In the cases of 5.1: Interactivity.Type and 5.5: Intended.End.User.Role it is less clear how to expand the vocabularies. These seem more openended. Hence, we recommend that, at a minimum, these fields be changed to open vocabularies until better consensus on an adequate term set can be obtained with the help of the various communities expected to be using the LOM. (We encourage readers of this paper to contribute to this further development.)

\section{Structural Issues}

In some cases we felt that the vocabulary should be replaced with a structured description. This was actually the case for 5.1: Interactivity.Type and 5.5: Intended.End.User.Role (see next section), as well as 5.7: Typical.Age. Range. Concerning the latter, K-12 educational resources in the United States are almost always referenced by grade level rather than age range. Other applications may require other measures, for example, the military needs to access resources by rank. Anticipating the need for flexibility, we recommended that 5.7: Typical.Age.Range be changed to a structured element with 5.7.1 Measure (e.g., 'Chronological Age', 'GEM Grade', etc.) and 5.7.2: Value (e.g., ' 12', '7-8', etc.).

More problematic are ways in which the value of one element depends on another. We noted that 5.9: Typical.Learning.Time depends on 5.7: Typical. Age.Range, for example, a textbook might be described as suitable for a fast paced graduate course or a two-semester undergraduate sequence. Erik Duval later pointed out that this applies to 5.4: Semantic.Density and 5.8: Difficulty as well. Hence, we recommended reorganizing these elements in the following manner: 
5.x Challenge. Level, consisting of one or more 4-tuples:

5.x.1 Educational. Level (formerly 5.7), consisting of one or more pairs:

5.x.1.1 Measure (e.g., Age, US Grade,...)

5.x.1.2 Value (e.g., 7-8)

5.x.2 Semantic. Density (formerly 5.4 )

5.x.3 Difficulty (formerly 5.8)

5.x.4 Learning Time (formerly 5.9)

Then, one could create multiple instances of 5.x: Challenge.Level, with the values of 5.x.2 through 5.x.4 being dependent on the value of 5.x.1: Educational. Level. It is possible to implicitly achieve the same effect by replicating entire LOM meta-data instances, one for each developmental level (or age); but we feel that it would be far more perspicuous and efficient to acknowledge the dependency explicitly in a structure such as the above.

\section{Our Extensions to the LOM}

The following extensions were made using 9: Classification.

\section{Audience}

This extension effectively replaces 5.5: Intended.End.User.Role with the GEM Audience (http://www.geminfo.org/Workbench/Metadata/Vocab_Audience. html), a two-part classification consisting of ToolFor (who uses the tool) and Beneficiary (who benefits). For example, a professional development resource that helps teachers handle learning disabled children in their classes is for the teacher but benefits the particular population of learning disabled students. We would prefer that 5.5: Intended.User.Role be modified to be composed of 5.5.1: Tool.For and 5.5.2: Beneficiary.

\section{Community Involvement}

This extension describes how a resource interacts with various stakeholders. We are designing this classification ourselves. We are considering a two-part classification. One for the community entity involved, and the other for the type of involvement.

\section{Discipline}

This extension describes the subject matter area covered by the resource. There is presently no LOM field that does this (other than possibly 1.7: Coverage, which has limitations beyond the scope of this paper). We are using 
the GEM Subject. This is a two-level classification system, requiring a twostep Taxon Path, for example, Science/Astronomy. We found it necessary to add two first-level classifications to the GEM Subject: Technology and Culture. An example using these subjects is shown in Figure 2, a partial screen dump of our Filemaker prototype implementation. We also needed a way to indicate cross-curricular integration. For this we again elected to modify the GEM taxonomy by allowing any major level Subject header to be listed as a minor header under the subject with which it is integrated. For example, Science/Mathematics would indicate that the resource integrates

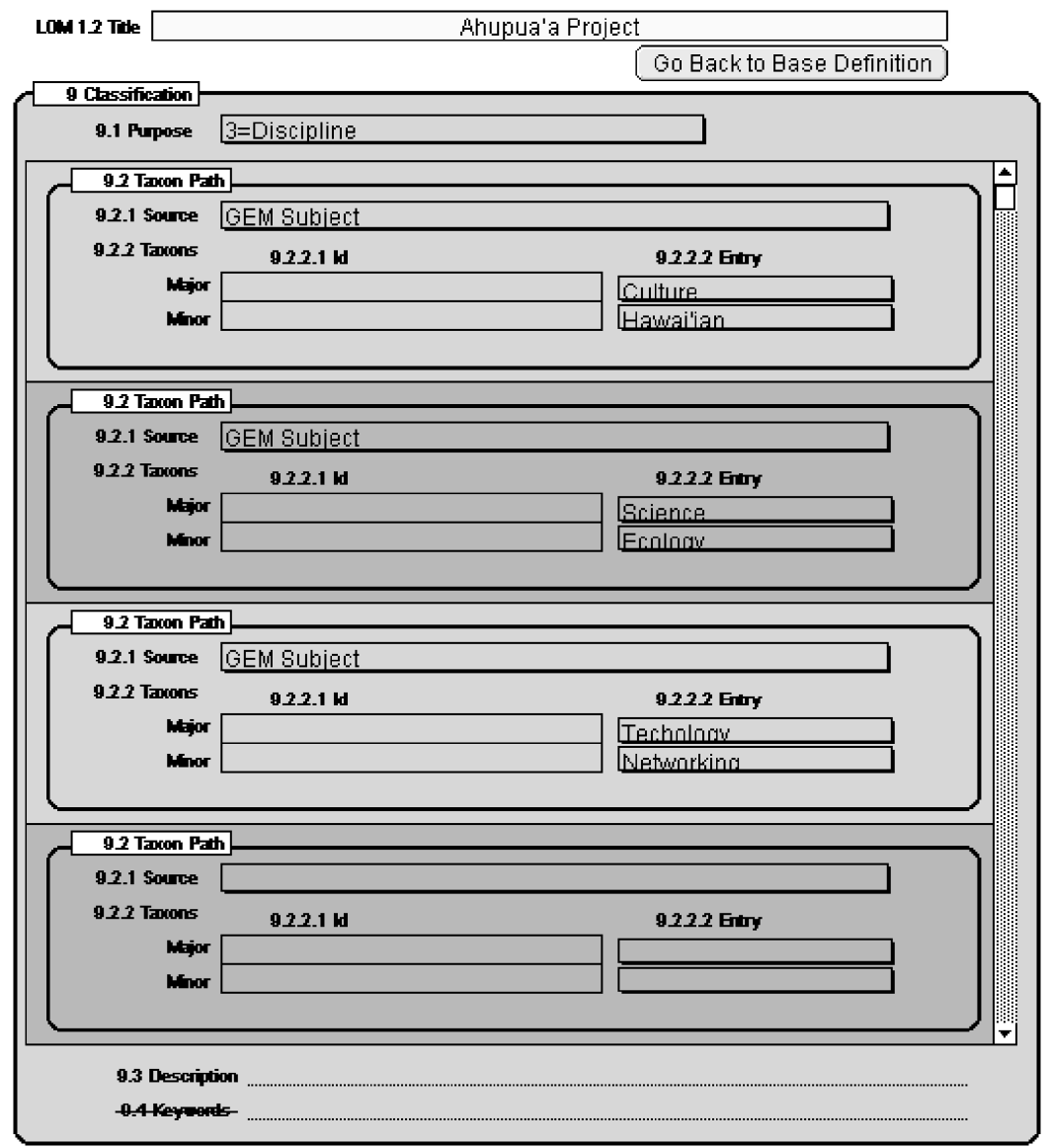

Fig. 2. Prototype HNLC Resource Database: a Discipline classification. 
Mathematics into Science. See http://www.geminfo.org/Workbench/Metadata/Vocab_Subject.html for the GEM Subject controlled vocabulary.

\section{Educational Level}

This extension augments LOM 5.7: Typical.Age.Range, and is structured as described in the previous section.

\section{Educational Objectives}

This extension addresses content and performance standards. It is distinct from Discipline because it is more specific: it aligns the resource with the particular standards that the resource is intended to help achieve. Examples of national (US) content and performance Standards include America's Choice (http://www.ncee.org/ac/intro.html); NCTM standards for mathematics (National Council of Teachers of Mathematics, http://nctm.org/standards/); the NSES for science (National Science Education Standards, http://www. nap.edu/readingroom/books $/ \mathrm{nses} / \mathrm{html} /$ ), and the National Educational Technology Standards (NETS http://cnets.iste.org/). An example of a state standard is the HCPS - Hawai i Content and Performance Standards (http://www. hcps.k12.hi.us/).

\section{Pedagogy}

This extension addresses the severe deficiency of the LOM's 5.1: Interactivity. Type, a closed vocabulary of active, expositive, mixed, undefined. We have recommended that the vocabulary for 5.1 be reopened. However, a rich description of interactivity is already available in the GEM Pedagogy-controlled vocabulary (http://www.geminfo.org/Workbench/Metadata/Vocab_ Pedagogy.html). This vocabulary has three facets: Teaching Methods (GEM provides a large vocabulary), Grouping (individual, small group, large group, etc.), and Assessment. Grouping is needed, for example, to distinguish learning objects intended for individual, collaborative, and classroom activities. Assessment is included because current approaches to pedagogy include assessment as part of the learning process (not just a measurement made after the fact). Students may even be provided with assessment rubrics to guide their own learning and to assess themselves and each other (Toth et al., 2001).

\section{CONCLUSIONS}

Internet technology for learning, including groupware and remote sensing, have the potential to bring teachers and students together with a greater 
diversity of human, natural and technological resources than was previously possible. Additionally, the current emphasis on systemic reform in public school education in the United States is encouraging and compelling a greater diversity of stakeholders to collaborate in their mutual interest in supporting achievement of high standards in the schools. These forces require that educators and their partners be aware of the resources that are potentially available to them and to understand the significance or potential utility of these resources with respect to educational objectives.

The HNLC Resource Database is being designed to meet such a need in the context of systemic standards-based reform in the state of Hawai i. The demands on such a database are high: it should interoperate with other major repositories, adequately describe a diversity of resources, yet relate them all to common content and performance standards and generally describe the resources in terms understandable to educators. The LTSC's LOM is being developed in part to lay the foundations for meeting such needs.

In this paper, we described our attempt to use the LOM for the HNLC Resource Database. We found that it provides a solid foundation in the form of many well thought out data elements as well as a means for extension. We also found that the LOM does not address all the needs of the HNLC Resource Database. This cannot be expected as the LOM is being designed to serve a variety of applications in government and industry as well as public education. We were able to deal with most of the limitations through the Classification method of extension. However, some of these extensions were due to premature closure of the LOM vocabularies. More problematic were structural dependencies between LOM elements that are not well captured at present. These issues were illustrated with examples from K-12 education.

The LOM standard is under active development at this writing. It is hoped that this paper will help increase awareness within the primary/secondary education sector worldwide of the LOM standards effort, and encourage your contribution to further development of the standard to be more appropriate for primary/secondary education needs. Anyone may participate: see http:// ltsc.ieee.org/ for details. We also recommend that special communities, such as the US K-12 education community, form their own working groups to build upon and extend the LOM as needed.

\section{ACKNOWLEDGEMENTS}

Thanks to David Nickles for contributing to the design of the HNLC meta-data and for implementing the first prototype of our resource database, and Laura 
Girardeau for constructing meta-data. Thanks to Erik Duval, Wayne Hodgins, Tom Murray, Brendon Towle, and Tom Wason for their 'meta-comments' on my LOM commentary. This work was funded in part by a development grant from the National Science Foundation's Rural Systemic Initiative.

\section{REFERENCES}

IEEE Std. 1484.12 (draft). Draft 4.1 of the Learning Objects Metadata (LOM), IEEE, Piscataway, NJ, USA, 2001. (Available: http://ltsc.ieee.org/doc/wg12/LOM_WD4.htm)

Koedinger, K., Suthers, D., \& Forbus, K. (1999). Component-based construction of a science learning space. International Journal of Artificial Intelligence in Education, 10, 292313. (Also available at http://cbl.leeds.ac.uk/ijaied/home.html)

Miller, P. (2000). Interoperability: What is it and why should I want it? Ariadne 24. (Available: http://www.ariadne.ac.uk/issue24/interoperability/)

Milstead, J., \& Feldman, S. (1999a). Metadata: Cataloging by any other name. Online, 23(1), 24-31. (Available: http://www.onlineinc.com/onlinemag/OL1999/milstead1.html)

Milstead, J., \& Feldman, S. (1999b). Metadata: Projects \& standards. Online, 23(1), 32-40. (Available: http://www.onlineinc.com/onlinemag/OL1999/milstead1.html\#projects)

Roschelle, J., DiGiano, C., Koutlis, M., Repenning, A., Phillips, J., Jackiw, N., \& Suthers, D. (1999). Developing educational software components. Computer, 32(9), 50-58. Piscataway, NJ: IEEE Computer Society.

Toth, E., Suthers, D., \& Lesgold, A. (2001, to appear). Mapping to know: The effects of evidence maps and reflective assessment on scientific inquiry skills. Science Education.

Wason, T. D. (unpublished). Dr. Tom's Meta-Data Primer. (Available: http://www.imsproject. org/drtommeta.html) 